\title{
Estudio de propiedades mecánicas locales de uniones soldadas por fricción agitación (FSW) en AA6061-T6 mediante Small Punch Test (SPT)
}

\section{Estimation of local mechanical properties in friction stir welded AA6061-T6 by Small Punch Test (SPT)}

\author{
Nelson Alvarez Villar ${ }^{1}$, Víctor Fierro ${ }^{1}$, Leonardo Nicolás Tufaro ${ }^{2}$, \\ Hernán Gabriel Svoboda ${ }^{3}$, Andrea Ansaldi ${ }^{1}$, \\ Rodrigo Gabarain ${ }^{1}$, Esteban Wenger ${ }^{1}$
}

\footnotetext{
${ }^{1}$ Departamento Mecánica Aplicada, CITEDEF, Juan B. de La Salle 4397, Villa Martelli, Buenos Aires, Argentina. e-mail: nalvarezvillar@ citedef.gob.ar

${ }^{2}$ Instituto Nacional de Tecnología Industrial, Centro de Investigación y Desarrollo en Mecánica, Av. Gral Paz 4999, San Martín, Buenos Aires, Argentina.

e-mail: ltufaro@inti.gob.ar

${ }^{3}$ GTSyCM3, INTECIN, Facultad de Ingeniería, Universidad de Buenos Aires - CONICET, Av. Las Heras 2214, Buenos Aires, Buenos Aires, Argentina.

e-mail: hsvobod@fi.uba.ar
}

\section{RESUMEN}

La Soldadura por Fricción Agitación (FSW) es un proceso en fase sólida que ha generado un fuerte impacto tecnológico en la soldadura de aleaciones de aluminio. Entre sus ventajas, se puede incluir la capacidad de unir materiales que son difíciles de soldar por fusión. Durante la soldadura de las aleaciones de aluminio tratables térmicamente, se observa la degradación de las propiedades metalúrgicas, obteniéndose menor resistencia en la soldadura que en el metal base. Los parámetros de soldadura definen las propiedades mecánicas de la unión. Los ensayos de Punzonado de Mini -Probetas (SPT) permiten el análisis de las propiedades locales en las zonas de soldadura, debido a que se requieren probetas de pequeñas dimensiones. En este trabajo, se prepararon dos lotes de probetas, con diferentes parámetros de soldadura y se estudiaron, mediante ensayos SPT, las propiedades de las diferentes zonas en soldaduras FSW. El metal base seleccionado fue chapa de aluminio AA6061-T6, con espesor de $3 \mathrm{~mm}$. Se registraron los ciclos térmicos, la carga axial y otros parámetros de soldadura. A partir de probetas extraídas de los cupones soldados, las soldaduras se caracterizaron mecánica y metalúrgicamente con ensayos convencionales, para su comparación con los resultados del SPT. El SPT aparece como una herramienta conveniente para la evaluación de propiedades mecánicas locales aplicable a uniones soldadas por FSW.

Palabras clave: Friction Stir Welding, Small Punch Test, propiedades mecánicas locales.

\begin{abstract}
Friction Stir Welding (FSW) is a solid state process that has reach a strong technological impact in welding of aluminum alloys. Among its advantages, it may include the ability of joining materials that are difficult to weld by fusion. In welding of heat treatable aluminum alloys, some degradation in metallurgical properties is observed, so welds show lower strength than base metal. Welding parameters define the mechanical properties of the welded joints. Small Punch Tests (SPT) allow the analysis of local properties in weld zones because small specimens are required. In this paper, two batches of samples were prepared with different welding parameters and local mechanical properties were studied by SPT tests. An AA6061-T6 sheet with thickness $3 \mathrm{~mm}$ was selected as base metal. Thermal cycle, axial load and another welding parameters were fully recorded. From samples taken from welded coupons, weldments were characterized mechanically and metallurgically by conventional tests for comparison with SPT results. SPT is a valuable tool to estimate local mechanical properties in FSW welds.
\end{abstract}


Keywords: Friction Stir Welding, Small Punch Test, local mechanical properties

\section{INTRODUCCIÓN}

Las aleaciones de aluminio de la serie 6XXX pueden soldarse mediante procesos como GTAW (TIG) y GMAW (MIG), produciendo soldaduras de buena calidad. Sin embargo, al ser aleaciones tratables térmicamente, debe tenerse en cuenta que el aporte térmico durante la soldadura en general provocará una disminución de las propiedades mecánicas en la Zona Afectada por el Calor (ZAC) y la Zona de Fusión (ZF), correspondiente al material fundido en el proceso como reportan AMBRIZ y MAYAGOITIA en [1]. Las propiedades mecánicas locales están relacionadas íntimamente con la microestructura que derivan de las transformaciones termomecánicas sufridas en cada zona característica de la unión durante el proceso de soldadura (crecimiento de grano, precipitación) estudiadas por ASTARITA et al. [2]. En la soldadura de estas aleaciones de aluminio, las propiedades mecánicas de cada zona de la unión presentan significativas diferencias, tal que los modos de falla observados dependen en gran medida de las propiedades mecánicas locales de acuerdo con LIU et al. [3].

La Soldadura por Fricción-Agitación (FSW) es un proceso en estado sólido aplicable a distintos materiales que viene siendo utilizado en forma creciente en distintas industrias como se detalla por MISHRA y MA en [4]. Este proceso ofrece ventajas significativas en relación con los procesos de soldadura por fusión. Entre ellas se incluyen una mayor eficiencia en la unión (en términos de resistencia a la tracción) y una mayor resistencia a la fatiga, especialmente para aleaciones de aluminio de acuerdo con THREADGILL et al. [5]. Por otra parte, no se requieren insumos y se logra una buena repetitividad en procesos. En consecuencia, en los últimos años se estudia la aplicación de FSW a la soldadura de distintos materiales (aún la unión de materiales disímiles, donde las ventajas de una soldadura en estado sólido son aún más significativas) como RODRÍGUEZ indica en [6].

En una soldadura FSW se distinguen zonas características: a) Zona Agitada (ZA, Nugget); b) Zona Afectada Termomecánicamente (ZATM); c) Zona afectada por el Calor (ZAC); d) Metal Base (MB). (Ver Figura 1).

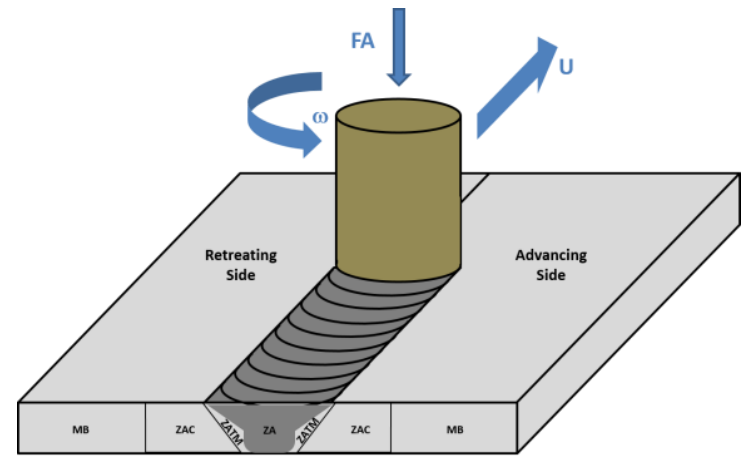

Figura 1: Esquema de zonas y parámetros de soldadura FSW.

Los parámetros de soldadura, tales como carga axial de la herramienta $(\mathrm{CA})$, velocidad rotacional $(\omega)$ y velocidad de avance (v), entre otros, determinarán las propiedades mecánicas de la unión como se reporta por MOREIRA et al. [7], a partir de las propiedades mecánicas locales en la soldadura. Dadas las características de la unión (metal base, tipo de junta y espesor), es posible identificar un conjunto de parámetros que optimicen las propiedades conforme a los requerimientos de diseño.

La microdureza es la técnica más habitual para estimar propiedades mecánicas locales. El perfil de microdureza revela la disminución de las propiedades en la ZATM y la ZAC con respecto al MB de acuerdo a lo estudiado por LEON y JAYAKUMAR [8]. Sin embargo, para caracterizar las propiedades mecánicas locales se recurre a diversas técnicas, incluyendo ensayos de tracción con probetas miniatura, ya que los ensayos convencionales no están disponibles, debido al pequeño tamaño de las zonas de estudio.

Los ensayos SPT están siendo desarrollados para aplicaciones tales como la caracterización mecánica del material de componentes y estructuras en servicio (por su característica de ensayo no destructivo) así como para determinar propiedades mecánicas locales (cuando no se pueden obtener probetas a ensayar con métodos convencionales) como indican RODRÍGUEZ et al. en [9]. En este trabajo utilizamos probetas de reducidas dimensiones (p. ej. $10 \times 10 \mathrm{~mm}^{2}$ con espesores de $0.5 \mathrm{~mm}$ ) tal que puede considerarse un ensayo no destructivo. 
En los últimos años, se extiende la utilización del Ensayo de Punzonado de Miniprobetas (SPT) para estimar localmente tanto la tensión de fluencia $S_{Y}$ como la resistencia mecánica $S_{U T S}$. Las propiedades mecánicas de zonas de la soldadura (tensión de fluencia $S_{\mathrm{Y}}$ y resistencia mecánica $S_{U T S}$ ) pueden estimarse en tanto la zona bajo análisis tenga una extensión de al menos $4 \mathrm{~mm}$ (que es la zona donde se produce la deformación hasta la rotura en los ensayos).

En los ensayos SPT se obtienen curvas carga (F) vs desplazamiento (u) tal como se esquematiza en la Figura 2. Sobre la curva se determinan $\mathrm{F}_{\mathrm{Y}}$, carga límite de la zona elástica y $\mathrm{F}_{\max }$, la carga máxima. A partir de esas cargas, están disponibles correlaciones para obtener estimación de las propiedades de tracción.

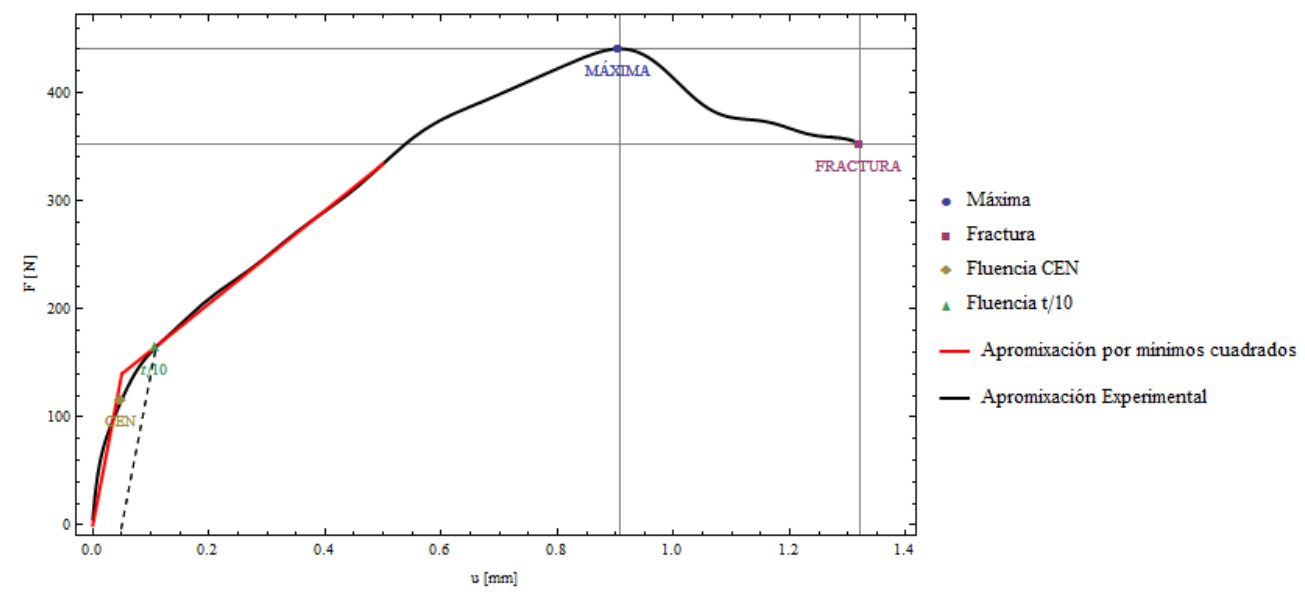

Figura 2: Esquema de las curvas experimentales obtenidas en ensayos SPT.

El objetivo de este trabajo es estudiar la aplicación de ensayos SPT para el análisis de las propiedades mecánicas locales de una unión soldada por FSW, complementándose con otras técnicas de caracterización mecánica y metalúrgica.

\section{MATERIALES Y MÉTODOS}

Como material base se utilizó una aleación de aluminio AA6061-T6 laminada y tratada con envejecimiento artificial. Esta aleación se considera soldable, aunque eventualmente es necesario realizar un tratamiento térmico posteriormente a la soldadura para recuperar las propiedades características del tratamiento T6.

La microdureza Vickers promedio (HV $1 \mathrm{~kg}$ ) del material base, medida sobre un corte transversal, fue de 113 HV. En la Tabla 1 se muestran las propiedades mecánicas en tracción del material base en la dirección longitudinal y transversal (promedio de 3 probetas ensayadas). Para esto se utilizó una máquina universal de ensayos Tinuis Olsen $5 \mathrm{~K}$ y las probetas obtenidas fueron de $3 \mathrm{~mm}$ de espesor, $12,5 \mathrm{~mm}$ de ancho y una longitud calibrada de $50 \mathrm{~mm}$, conforme a la norma ASTM E8M. Se obtuvieron valores similares para ambas direcciones.

Tabla 1: Propiedades mecánicas del MB comparado con las especificaciones de la Aluminum Association para el AA6061-T6.

\begin{tabular}{c|c|c|c|c}
\hline DESIGNACIÓN & $\begin{array}{c}\mathbf{S}_{\mathbf{Y}} \\
{[\mathrm{MPa}]}\end{array}$ & $\begin{array}{c}\mathbf{S}_{\text {UtS }} \\
{[\mathrm{MPa}]}\end{array}$ & $\begin{array}{c}\text { ALARGAMIENTO } \\
\text { A ROTURA [\%] }\end{array}$ & $\begin{array}{c}\text { TIPO DE } \\
\text { LA FRACTURA }\end{array}$ \\
\hline MB Longitudinal & $311 \pm 2$ & $363 \pm 2$ & $14,3 \pm 0,8$ & Dúctil $45^{\circ}$ \\
\hline MB Transversal & $307 \pm 1$ & $363 \pm 2$ & $15,2 \pm 0,5$ & ${\text { Dúctil } 45^{\circ}}^{\circ}$ \\
\hline AA 6061-T6 típicos & 276 & 310 & 12 & \\
\hline
\end{tabular}

En la soldadura por FSW se prevé que incrementando la relación $\omega / \mathrm{v}$, también se incrementan el aporte de calor y la agitación en la ZA como indican TUFARO y SVOBODA en [10]. Para este trabajo se soldaron chapas de espesor $3 \mathrm{~mm}$ en cupones de dimensiones 150 x $150 \mathrm{~mm}$, con dos configuraciones de parámetros. Se varió la velocidad de avance v, definiendo las dos condiciones: a) Probetas 680-51 (v = $51 \mathrm{~mm} / \mathrm{min})$ y b) Probetas 680-206 ( $\mathrm{v}=206 \mathrm{~mm} / \mathrm{min})$. En ambos casos se mantuvo constante la velocidad angular $\omega(680 \mathrm{rpm})$. El ángulo de inclinación fue de 1,5 y se utilizó una herramienta de acero H13 de 
hombro cóncavo y pin cónico con tres planos a $120^{\circ}$. El equipo utilizado para la soldadura y el proceso se describen en un trabajo anterior de TUFARO et al. [11].

De cada condición de soldadura se extrajeron cortes transversales que se prepararon para observación metalográfica. Se analizaron macrográficamente para identificar las zonas características en la unión y determinar la presencia de defectos.

Para determinar cualitativamente diferencia en las propiedades mecánicas, en probetas obtenidas para cada unión, se tomaron perfiles de microdureza, a una profundidad de $1 \mathrm{~mm}$ respecto de la cara sobre la que actuó la herramienta. Al mismo tiempo, así se indica la extensión de las distintas zonas que constituyen la soldadura. Se utilizó un microdurómetro EMCO Test Durascan 10 y los ensayos se realizaron conforme a ASTM E384.

Para la determinación de las propiedades en tracción se extrajeron 2 probetas transversales de cada condición de soldadura con las mismas dimensiones mencionadas anteriormente de acuerdo a la norma ASTM E8M (Figura 3).

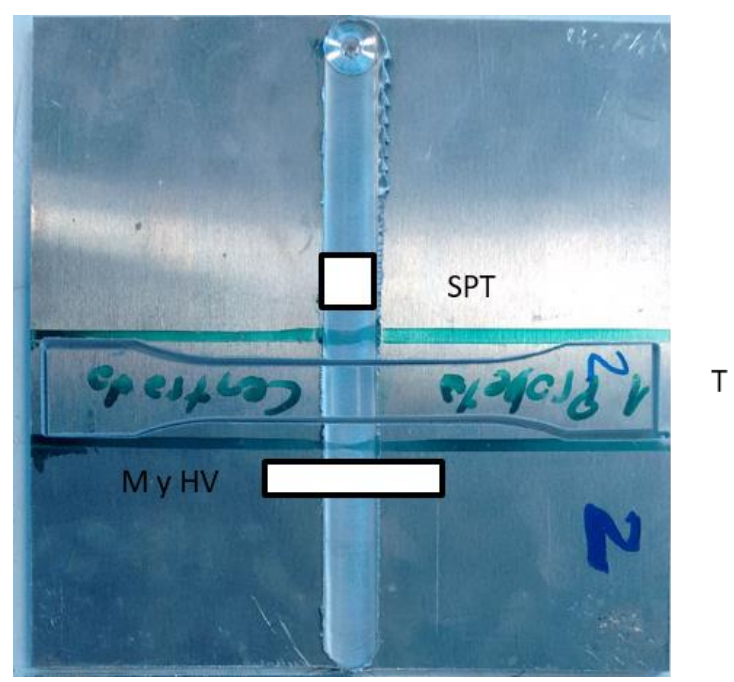

Figura 3: Probeta soldada por FSW. Extracción probetas de tracción y de SPT.

Diversos autores han propuesto expresiones para relacionar las propiedades mecánicas de tracción locales con la microdureza medida. Entre ellos, STATHERS [12] propone distintas relaciones para el MB (y la ZAC), que, en este trabajo, también aplicamos a la ZA:

$$
\begin{aligned}
& S_{y}=3,6337 \cdot H V-94,949 \\
& S_{U T S}=2,4848 \cdot H V+44,32
\end{aligned}
$$

donde HV es la microdureza Vickers, tal que la expresión permite obtener las tensiones en MPa.

De los resultados obtenidos en los ensayos de caracterización mecánica y metalúrgica, se explica que al aumentar la velocidad de avance, se espera una menor degradación metalúrgica por ser menor el aporte de energía. En consecuencia, la ZA se espera que tenga mayor dureza y resistencia y menor ductilidad al soldar con $\mathrm{v}=206 \mathrm{~mm} / \mathrm{min}$.

Finalmente, se preparon probetas SPT de la ZA, de $10 \times 10 \mathrm{~mm}^{2}$ (Figura 3) con un espesor nominal de 0,5 mm, para comparar las propiedades de la ZA (Figura 3) en función de la velocidad transversal v (para una FA y una $\omega$ dadas) y con el metal base MB. Se prepararon tres grupos, siguiendo los procedimientos indicados para obtener terminación superficial necesaria sin afectar las propiedades mecánicas. Este procedimiento y la descripción de los ensayos siguen las recomendaciones del CEN (European Committee for Standarization) detalladas en el draft CWA 15627. Para la designación de las probetas SPT, se utilizó MBXX para las probetas del metal base, CIXX y CIIXX para las de los cordones correspondientes a las probetas 680-51 y 680-206, respectivamente. En los ensayos se utilizó un dispositivo adaptado a una máquina SINTECH 2/DL, se aplicó una carga hasta la rotura con una velocidad de $1 \mathrm{~mm} / \mathrm{min}$ y se registró continuamente carga $\mathrm{F}$ y desplazamiento $\mathrm{u}$.

Para normalizar las cargas $F_{Y}$ y $F_{\max }$ seguimos las expresiones (3), (4), (5) y (6) indicadas por 
GARCÍA [13]:

$$
\begin{aligned}
& S_{y(1)}=a_{1} \cdot \frac{F_{y(C E N)}}{h^{2}} \\
& S_{y(2)}=a_{2} \cdot \frac{F_{y(h / 10)}}{h^{2}} \\
& S_{U T S(1)}=b_{1} \cdot \frac{F_{\max }}{h} \\
& S_{U T S(2)}=b_{2} \cdot \frac{F_{\max }}{u_{\max } \cdot h}
\end{aligned}
$$

donde $\mathrm{h}$ es el espesor de la probeta, $\mathrm{F}_{\mathrm{Y}(\mathrm{CEN})}$ y $\mathrm{F}_{\mathrm{Y}(\mathrm{h} / 10)}$ son las cargas de transición elástico-plástica, $\mathrm{F}_{\max }$ la carga máxima y $\mathrm{a}_{1}, \mathrm{a}_{2}, \mathrm{~b}_{1}$ y $\mathrm{b}_{2}$ son constantes del ensayo.

\section{RESULTADOS}

\subsection{Caracterización Macrográfica}

En las Figuras 4 y 5 se muestran las macrografías de las probetas soldadas por FSW con velocidad de avance de 51 y $206 \mathrm{~mm} / \mathrm{min}$, respectivamente.

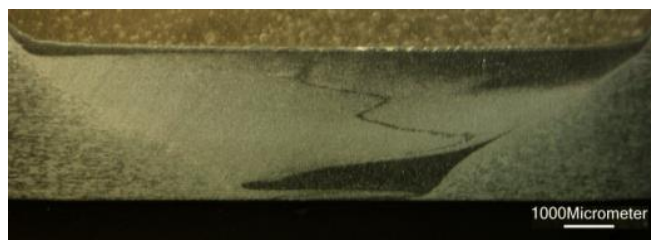

Figura 4: Macrografía de la probeta soldada 680-51.

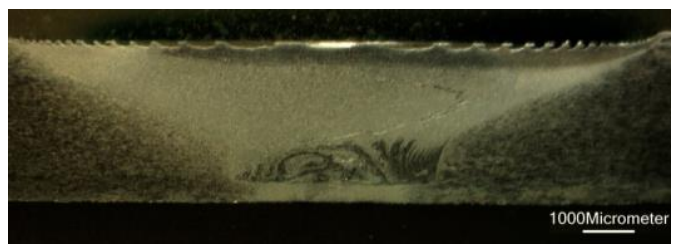

Figura 5: Macrografía de la probeta soldada 680-206.

En la Figura 4 (probetas 680-51) se observa una mayor ZA que en la Figura 5 (probetas 680-260), lo cual está asociado al mayor aporte de calor. En general no se observan defectos macroscópicos para ninguna de las dos condiciones de soldadura. En ambos casos se observa la línea de óxidos remanente en zig-zag en el centro de la zona agitada, asimismo, en la parte inferior de la ZA se puede ver un patrón del tipo aros de cebolla, habitualmente observado por MISHRA [14]. En la macrografía de las probetas 680-206 no se observan defectos macroscópicos, indicando que sería posible aumentar la velocidad de avance a valores mayores que $206 \mathrm{~mm} / \mathrm{min}$ para reducir el aporte térmico y la degradación metalúrgica. Sin embargo, se debe considerar que, al aumentar la velocidad de avance, el flujo plástico en la base del pin disminuye, lo que podría provocar una falta de penetración si no se modifica levemente la longitud del pin de la herramienta.

\subsection{Perfiles de microdureza Vickers (HV)}

En la Figura 6 se observan los perfiles de microdureza Vickers obtenidos. Puede verse que la microdureza disminuye desde el material base hacia el centro de la ZA. La microdureza mínima del perfil se encuentra en la ZA/ZTMA/ZAC caliente, siendo el valor promedio de dicha zona de 56 y $61 \mathrm{HV}$ para las probetas $680-51$ y 680-206, respectivamente. Además de la mayor microdureza en la zona agitada, se observa una menor extensión para la zona degradada en la unión soldada con $206 \mathrm{~mm} / \mathrm{min}$, lo cual puede atribuirse al menor aporte de calor, de acuerdo a lo mencionado anteriormente. El índice de dureza $\left(\mathrm{I}_{\mathrm{H}}\right)$ definido como el 
cociente entre la dureza mínima y la dureza del MB, fue de 0,50 y de 0,54 para las probetas 680-51 y 680-206, respectivamente.

Para la caracterización de las zonas de la soldadura (conforme a las Figuras 4 y 5) se pueden requerir la realización de perfiles de microdureza a distintas distancias de la superficie.

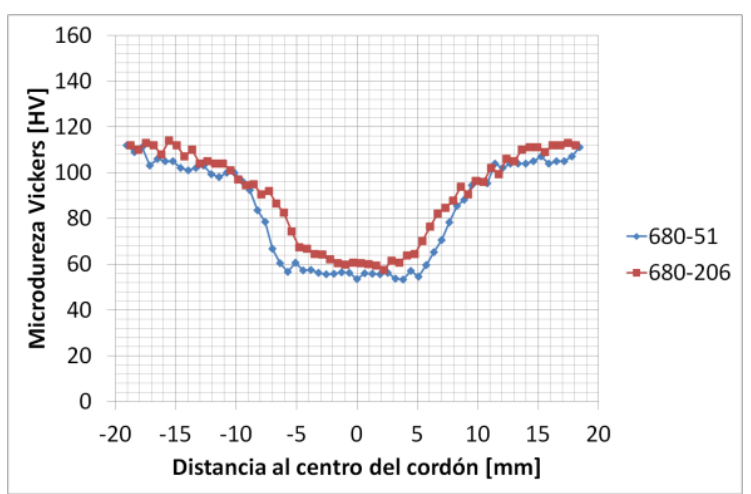

Figura 6: Perfil de microdureza HV $1 \mathrm{~kg}$.

\subsection{Ensayos de tracción}

En la Tabla 2 se reportan las propiedades mecánicas en tracción de las uniones soldadas (promedio de dos probetas ensayadas conforme ASTM E8M).

Tabla 2: Propiedades mecánicas de las uniones soldadas.

\begin{tabular}{c|c|c|c|c|c}
\hline DESIGNACIÓN & $\begin{array}{c}\mathbf{S}_{Y} \\
\text { [MPa] }\end{array}$ & $\begin{array}{c}\text { EFICIENCIA DE } \\
\text { JUNTA [\%] }\end{array}$ & $\begin{array}{c}\text { ALARGAMIENTO } \\
\text { A ROTURA [\%] }\end{array}$ & $\begin{array}{c}\text { UBICACIÓN DE } \\
\text { LA FRACTURA }\end{array}$ & $\begin{array}{c}\text { TIPO DE } \\
\text { LA FRACTURA }\end{array}$ \\
\hline $680-51$ & $186 \pm 1$ & $51 \pm 1$ & $8,1 \pm 0,2$ & Zona Agitada & Dúctil $45^{\circ}(\mathrm{ZA})$ \\
\hline $680-206$ & $207 \pm 7$ & $57 \pm 2$ & $6,2 \pm 0,8$ & Zona Agitada & Dúctil $45^{\circ}(\mathrm{ZA})$ \\
\hline
\end{tabular}

Se observa un aumento de la resistencia y disminución del alargamiento a rotura para la condición soldada con la mayor velocidad de avance, consistentemente con lo determinado en el perfil de microdureza, lo cual estaría asociado al menor aporte térmico, como se mencionó anteriormente.

Se tomó como referencia la resistencia del MB en la dirección transversal para el cálculo de la eficiencia de la unión, que se define como la relación entre la resistencia de la soldadura con respecto a la resistencia del MB. La eficiencia obtenida está en el orden de 50-60\%, que puede optimizarse a partir de la determinación de las propiedades mecánicas locales en la unión. La eficiencia de la soldadura tiene amplios márgenes de mejora, ya sea a través de la optimización de los parámetros como empleando un tratamiento térmico post-soldadura.

En todos los casos, la fractura se produjo en la ZA y en forma dúctil. Por lo tanto, la fractura se localiza en la zona de menor resistencia cuando no se observan defectos de soldadura. En la Figura 7 se muestran la ubicación y características de la fractura de las probetas de tracción de ambas condiciones analizadas.
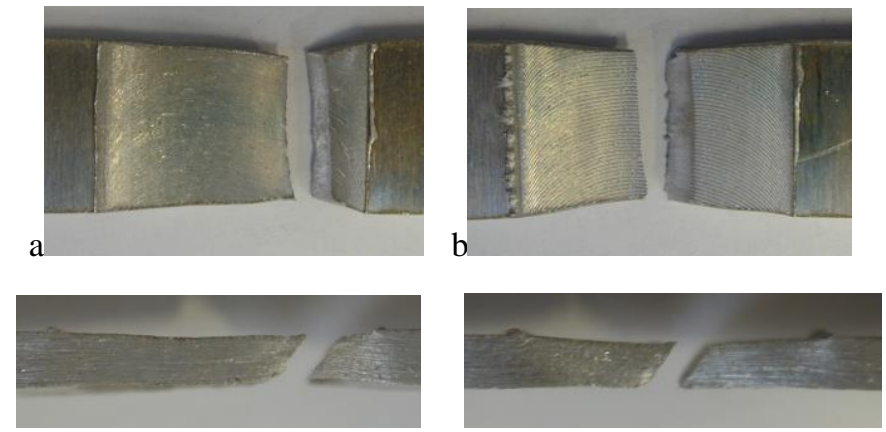

Figura 7: Fractura de las probetas de tracción: a-680-51, b-680-206. 
Tabla 3. Cargas y tensiones de fluencia y máxima para cada grupo de probetas con sus valores medios y desvío estándar. Los subíndices hacen referencia a los diferentes criterios considerados: $S_{\mathrm{Y}(1)}=\mathrm{a}_{1} \mathrm{~F}_{\mathrm{Y}(\mathrm{CEN})} /\left(\mathrm{h}^{\wedge} 2\right) ; \mathrm{S}_{\mathrm{Y}(2)}=\mathrm{a}_{2} \mathrm{~F}_{\mathrm{Y}(\mathrm{t} / 10)} /\left(\mathrm{h}^{\wedge} 2\right)$; $\mathrm{S}_{\mathrm{UTS}(1)}=\mathrm{b}_{1} \mathrm{~F}_{\max } / \mathrm{h} ; \mathrm{S}_{\mathrm{UTS}(2)}=\mathrm{b}_{2} \mathrm{~F}_{\max } /(\mathrm{dp} \mathrm{h})$.

\begin{tabular}{|c|c|c|c|c|c|c|c|c|}
\hline ID & $\begin{array}{c}\mathbf{h} \\
{[\mathrm{mm}]}\end{array}$ & $\begin{array}{c}F_{y} \text { (CEN) } \\
{[N]}\end{array}$ & $\begin{array}{c}F_{y(h / 10)} \\
{[N]}\end{array}$ & $\begin{array}{l}\mathbf{F}_{\max } \\
{[\mathrm{N}]}\end{array}$ & $\begin{array}{c}\mathbf{S}_{\mathrm{y}(1)} \\
{[\mathrm{MPa}]}\end{array}$ & $\begin{array}{c}S_{\mathrm{y}(2)} \\
{[\mathrm{MPa}]}\end{array}$ & $\begin{array}{r}\mathrm{S}_{\mathrm{uts}(1)} \\
{[\mathrm{MPa}]}\end{array}$ & $\begin{array}{c}\mathbf{S}_{\mathrm{uts}(2)} \\
{[\mathrm{MPa}]}\end{array}$ \\
\hline \multicolumn{9}{|c|}{ MB: metal base } \\
\hline MB11 & 0,48 & 118 & 168 & 436 & 330 & 320 & 343 & 362 \\
\hline MB7 & 0,50 & 88 & 133 & 449 & 225 & 234 & 340 & 360 \\
\hline MB8 & 0,50 & 120 & 171 & 457 & 308 & 301 & 345 & 369 \\
\hline MB9 & 0,50 & 119 & 170 & 473 & 307 & 298 & 357 & 339 \\
\hline MB13 & 0,50 & 105 & 159 & 440 & 271 & 278 & 333 & 336 \\
\hline MB16 & 0,50 & 121 & 171 & 457 & 313 & 301 & 345 & 350 \\
\hline MB17 & 0,50 & 114 & 163 & 459 & 294 & 287 & 346 & 354 \\
\hline MB18 & 0,50 & 117 & 168 & 453 & 300 & 295 & 342 & 325 \\
\hline MB19 & 0,50 & 116 & 165 & 441 & 299 & 289 & 333 & 318 \\
\hline MB20 & 0,50 & 108 & 155 & 428 & 278 & 272 & 323 & 346 \\
\hline MB14 & 0,51 & 124 & 178 & 453 & 307 & 301 & 336 & 346 \\
\hline MB15 & 0,51 & 81 & 126 & 474 & 200 & 212 & 351 & 342 \\
\hline MB21 & 0,51 & 90 & 139 & 457 & 223 & 235 & 338 & 318 \\
\hline MB10 & 0,52 & 123 & 176 & 445 & 294 & 286 & 323 & 325 \\
\hline MB12 & 0,52 & 99 & 150 & 450 & 235 & 244 & 327 & 304 \\
\hline \multicolumn{2}{|c|}{ Media } & $110 \pm 14$ & $160 \pm 16$ & $451 \pm 12$ & $279 \pm 39$ & $277 \pm 31$ & $339 \pm 10$ & $340 \pm 19$ \\
\hline \multicolumn{9}{|c|}{ Cordón I: ZA probetas $680-51$} \\
\hline CI5 & 0,44 & 37 & 54 & 375 & 123 & 122 & 322 & 189 \\
\hline CI9 & 0,46 & 43 & 60 & 386 & 131 & 124 & 317 & 186 \\
\hline $\mathrm{CI} 7$ & 0,47 & 49 & 67 & 388 & 142 & 133 & 312 & 192 \\
\hline CI8 & 0,47 & 42 & 61 & 370 & 123 & 122 & 297 & 181 \\
\hline $\mathrm{CI} 2$ & 0,49 & 45 & 71 & 409 & 121 & 129 & 315 & 193 \\
\hline CI4 & 0,49 & 61 & 79 & 475 & 162 & 145 & 366 & 205 \\
\hline CI10 & 0,51 & 42 & 66 & 409 & 104 & 111 & 303 & 180 \\
\hline CI1 & 0,52 & 54 & 79 & 447 & 129 & 128 & 325 & 191 \\
\hline $\mathrm{CI} 3$ & 0,52 & 46 & 74 & 453 & 111 & 121 & 329 & 217 \\
\hline CI6 & 0,52 & 45 & 66 & 444 & 108 & 107 & 323 & 188 \\
\hline \multicolumn{2}{|c|}{ Media } & $47 \pm 7$ & $68 \pm 8$ & $416 \pm 37$ & $126 \pm 17$ & $124 \pm 11$ & $321 \pm 19$ & $192 \pm 11$ \\
\hline \multicolumn{9}{|c|}{ Cordón II: ZA probeta $680-206$} \\
\hline CII11 & 0,49 & 58 & 86 & 486 & 154 & 157 & 375 & 237 \\
\hline CII12 & 0,52 & 69 & 98 & 524 & 165 & 158 & 380 & 237 \\
\hline CII13 & 0,50 & 54 & 84 & 518 & 140 & 147 & 391 & 234 \\
\hline CII14 & 0,50 & 60 & 89 & 486 & 155 & 157 & 367 & 220 \\
\hline CII15 & 0,49 & 61 & 89 & 515 & 164 & 162 & 397 & 260 \\
\hline CII16 & 0,50 & 60 & 89 & 525 & 155 & 156 & 397 & 246 \\
\hline \multicolumn{2}{|c|}{ Media } & $61 \pm 5$ & $89 \pm 5$ & $509 \pm 18$ & $156 \pm 9$ & $156 \pm 3$ & $385 \pm 12$ & $239 \pm 13$ \\
\hline
\end{tabular}

Este aspecto es de fundamental importancia, dado que los resultados de tracción serán empleados para calibrar las propiedades mecánicas locales de la ZA través de los ensayos SPT. En este sentido es 
fundamental que los valores obtenidos de los ensayos estén asociados a las características metalúrgicas de cada zona y no se encuentren afectados por defectos producidos durante la soldadura. Se observa que para ambas condiciones de soldadura, la fractura se produjo en la ZA y no se vio afectada por ningún tipo de defecto asociado a la soldadura FSW, al menos detectable mediante observación visual.

Asimismo, el índice de dureza $\mathrm{I}_{\mathrm{H}}$ alcanzó valores similares a la eficiencia de junta, para ambas condiciones de soldadura. En este sentido ambos índices evalúan las propiedades de la ZA, ya que allí se ubican la dureza mínima y la zona de rotura en el ensayo de tracción.

\subsection{Ensayos SPT}

En la Tabla 3 (ver página anterior) se reportan los resultados y los valores medios obtenidos para los ensayos SPT de las tres condiciones analizadas (MB, Cordón I y Cordón II).

De acuerdo con los procedimientos empleados se identificaron los puntos característicos de las curvas F-u obtenidas en los ensayos SPT (ver Figura 2) y se estimaron las propiedades de tracción, conforme se indica en la Tabla 3. En la Tabla 4 se comparan las propiedades mecánicas a la tracción en MB, Cordón I y Cordón II.

Tabla 4: Comparación de las cargas y tensiones obtenidas por el ensayo de micropunzonado para las probetas de metal base y los cordones de soldadura I y II.

\begin{tabular}{|c|c|c|c|c|c|c|c|}
\hline & $\begin{array}{c}\mathbf{F}_{\mathrm{Y}(\mathrm{CEN})} \\
{[\mathrm{N}]}\end{array}$ & $\begin{array}{c}\mathbf{F}_{\mathrm{Y}(\mathrm{h} / 10)} \\
{[\mathrm{N}]}\end{array}$ & $\begin{array}{c}\mathbf{F}_{\max } \\
{[\mathbf{N}]}\end{array}$ & $\begin{array}{c}\mathrm{S}_{\mathrm{Y}(1)} \\
{[\mathrm{MPa}]}\end{array}$ & $\begin{array}{c}\mathrm{S}_{\mathrm{Y}(2)} \\
{[\mathrm{MPa}]}\end{array}$ & $\begin{array}{l}\mathrm{S}_{\mathrm{UTS}(1)} \\
\text { [MPa] }\end{array}$ & $\begin{array}{l}\mathrm{S}_{\text {UTS (2) }} \\
\text { [MPa] }\end{array}$ \\
\hline MB SPT & 110 & 160 & 451 & $279 \pm 39$ & $277 \pm 31$ & $339 \pm 10$ & $340 \pm 19$ \\
\hline Referencia MB & & & & $311 \pm 2$ & $311 \pm 2$ & $363 \pm 2$ & $363 \pm 2$ \\
\hline Cordón I & 47 & 68 & 416 & $126 \pm 17$ & $124 \pm 11$ & $321 \pm 19$ & $192 \pm 11$ \\
\hline Cordón II & 61 & 89 & 509 & $156 \pm 9$ & $156 \pm 3$ & $285 \pm 12$ & $239 \pm 13$ \\
\hline
\end{tabular}

De los ensayos para el MB, los resultados son consistentes para la tensión de fluencia $S_{\mathrm{Y}}$ y la resistencia mecánica $S_{\text {UTS }}$ con respecto a los obtenidos por ensayos convencionales para el metal base. Se destaca que para la resistencia mecánica $S_{U T S}$, se obtienen mejores resultados con la expresión (6) que incluye, para la normalización, el valor de desplazamiento para la carga máxima además del espesor de la probeta.

Asimismo, puede verse que se mantiene la tendencia de los mayores valores de resistencia para la condición de mayor velocidad de soldadura.

\subsection{Simulación}

Para evaluar los ensayos SPT mediante simulación numérica se consideran factores que influyen en los resultados pero no pueden medirse directamente en los ensayos (entre ellos la fricción entre probeta y matriz, el efecto de la diferencia en el espesor de las probetas). En nuestro análisis no se considera daño mecánico, por lo que únicamente se obtienen simulaciones para la primera parte de las curvas P-u, donde evaluamos la tensión de fluencia $S_{Y}$.

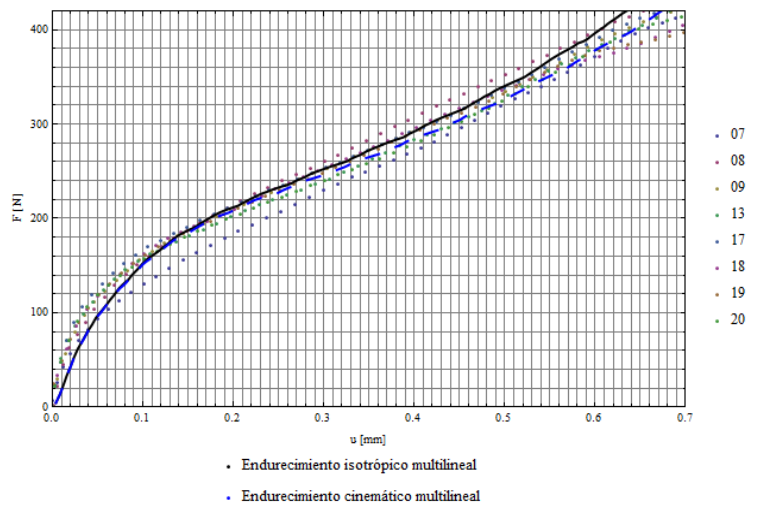

Figura 8: Comparación de curvas experimentales con las curvas obtenidas de la simulación computacional. 
Utilizamos el código ALGOR v12.0 de acuerdo con publicaciones anteriores, tratando el ensayo como un problema bidimensional axial simétrico. Para el material consideramos las alternativas: a) endurecimiento isotrópico multilineal (MISO) y b) endurecimiento cinemático multilineal (KIN). Ambos modelos de plasticidad permitirían aproximar la primera parte de la curva F-u, como se observa en la Figura 8.

En la Tabla 5 se informan los valores de $\mathrm{F}_{Y}$ para el MB, obtenidos mediante simulación numérica. Se consideraron probetas de espesor $\mathrm{h}=0,5 \mathrm{~mm}$ y fricción entre probeta y matriz $\mu=0,3$. La simulación se ajusta a los datos experimentales, que presentan una dispersión adecuada. Para evaluar la simulación, es importante la definición de la carga de transición $\mathrm{F}_{\mathrm{Y}}$ y el modelo de plasticidad utilizados. Los resultados obtenidos podrían extenderse del metal base a la ZA y eventualmente a la ZAC.

Tabla 5: Valores medios de $\mathrm{F}_{\mathrm{Y}}$ y su comparación con la simulación con dos modelos de plasticidad a) MISO y b) KIN.

\begin{tabular}{c|c|c}
\hline ID & $\begin{array}{c}\mathbf{F}_{\mathbf{y}(\mathbf{C E N})} \\
{[\mathbf{N}]}\end{array}$ & $\begin{array}{c}\mathbf{F}_{\mathbf{y}(\mathbf{h} / 10)} \\
{[\mathbf{N}]}\end{array}$ \\
\hline MB8 & 120 & 171 \\
\hline MB9 & 119 & 170 \\
\hline MB13 & 105 & 159 \\
\hline MB16 & 121 & 171 \\
\hline MB17 & 114 & 163 \\
\hline MB18 & 117 & 168 \\
\hline MB19 & 116 & 165 \\
\hline MB20 & 108 & 155 \\
\hline Experimental & $115 \pm 6$ & $165 \pm 6$ \\
\hline Simulación MISO & 142 & 193 \\
\hline Simulación KIN & 141 & 189 \\
\hline
\end{tabular}

En la Tabla 6 se comparan los valores medios para la tensión de fluencia $S_{Y}$ y la resistencia mecánica $\mathrm{S}_{\mathrm{UTS}}$ obtenidas por SPT, con las estimaciones derivadas del perfil de microdureza conforme a las expresiones (1) y (2).

Tabla 6: Cuadro comparativo entre resultados de ensayo de micropunzonado y las estimaciones por microdureza.

\begin{tabular}{|c|c|c|c|c|c|c|c|c|}
\hline & \multicolumn{2}{|c|}{ TRACCION } & \multicolumn{2}{|c|}{ MICRODUREZA } & \multicolumn{4}{|c|}{ SPT } \\
\hline & $\begin{array}{c}S_{Y} \\
{[\mathrm{MPa}]}\end{array}$ & $\begin{array}{c}\text { Suts } \\
{[\mathrm{MPa}]}\end{array}$ & $\begin{array}{c}\mathrm{S}_{\mathrm{Y}} \\
{[\mathrm{MPa}]}\end{array}$ & $\begin{array}{c}\text { Suts } \\
{[\mathrm{MPa}]}\end{array}$ & $\begin{array}{c}\mathrm{S}_{\mathrm{Y}(1)} \\
{[\mathrm{MPa}]}\end{array}$ & $\begin{array}{c}\mathrm{S}_{\mathrm{Y}(2)} \\
{[\mathrm{MPa}]}\end{array}$ & $\begin{array}{l}\text { Suts(1) }_{\text {(MPa] }} \\
\text { [MPa }\end{array}$ & $\begin{array}{l}S_{\text {UTS(2) }} \\
{[\mathrm{MPa}]}\end{array}$ \\
\hline MB & $311 \pm 2$ & $363 \pm 2$ & $219 \pm 14$ & $262 \pm 10$ & $279 \pm 39$ & $277 \pm 31$ & $339 \pm 10$ & $340 \pm 19$ \\
\hline Cordón I & - & $186 \pm 1$ & $112 \pm 4$ & $184 \pm 3$ & $126 \pm 17$ & $124 \pm 11$ & $321 \pm 19$ & $192 \pm 11$ \\
\hline Cordón II & - & $207 \pm 7$ & $129 \pm 1$ & $197 \pm 1$ & $156 \pm 9$ & $156 \pm 3$ & $285 \pm 12$ & $239 \pm 13$ \\
\hline
\end{tabular}

Para el MB, la aproximación mediante microdureza provee resultados menores que los medidos mediante en ensayo de tracción, mientras que los resultados de SPT, presentan un mejor acuerdo, tanto respecto del límite de fluencia como de la resistencia a la tracción. Para la ZA de las uniones soldadas por FSW, los resultados de la microdureza y de SPT con la expresión (6) presentan una buena aproximación, presentando errores similares. En el caso de la microdureza, los resultados son algo inferiores que los de tracción, mientras que en el caso de los ensayos SPT son algo superiores.

Los resultados pueden considerarse aceptables para evaluar al SPT como una herramienta adecuada para la estimación de propiedades mecánicas locales.

\section{DISCUSIÓN}

En aleaciones base aluminio tratables térmicamente, las propiedades mecánicas locales (especialmente tensión de fluencia y alargamiento) varían sensiblemente con la forma y distribución de las segundas fases. Si bien se estiman las propiedades mecánicas dependen de la microestructura y esta se caracteriza usualmente mediante perfiles de microdureza. Sin embargo, no puede asegurarse que las propiedades mecánicas de tracción están relacionadas linealmente con la microdureza, y que ésta relación pueda aplicarse a las distintas zonas de la soldadura. 
Por las características de los ensayos SPT para la extracción de probetas, las propiedades mecánicas del material no se ven afectadas en la medida que se respeten los procedimientos para la preparación de las probetas y la realización de los ensayos. Asimismo, la caracterización mediante ensayos SPT podría proveer una estimación local, pero no tan puntual como la microdureza, lo que podría proveer un procedimiento en general más robusto.

Se verificaron diferencias significativas en las propiedades mecánicas del MB con la ZA, que se atribuyen a los parámetros de soldadura empleados.

\section{CONCLUSIONES}

Los ensayos SPT proporcionan una estimación de las propiedades locales de tracción que puede ser importante para predecir la distribución de tensiones y el comportamiento mecánico de las uniones.

La resistencia del material en las zonas de la soldadura puede variar atribuyéndose los cambios a la microestructura y la presencia de segundas fases. La tensión de fluencia y el alargamiento locales varían en un rango importante, por lo que el SPT aporta información para optimizar la selección de parámetros de soldadura y también puede utilizarse para definir la conveniencia de tratamientos térmicos posteriores.

El SPT aparece como una herramienta conveniente para la evaluación de propiedades mecánicas locales.

\section{AGRADECIMIENTOS}

Los autores agradecen al Ministerio de Defensa, ya que las actividades del proyecto fueron posibles al serle asignado el PIDDEF 16/12.

\section{BIBLIOGRAFÍA}

[1] AMBRIZ, R.R. ,MAYAGOITIA, V., "Welding of Aluminum Alloys, Recent Trends in Processing and Degradation of Aluminium Alloys, Prof. Zaki Ahmad (Ed.), ISBN: 978-953-307-734-5, 2001. InTech:http://www.intechopen.com/books/recent-trends-in-processing-and-degradation-of-aluminiumalloys/weldingof-aluminum-alloys

[2] ASTARITA, A., SQUILLACE, A., NELE, L. "Mechanical characteristics of welded joints of aluminum alloy 6061 T6 formed by Arc and Friction Stir Welding”, Metal Science and Heat Treatment, v. 57, pp. 9-10, 2016.

[3] LIU, H., FUJII, H., MAEDA, M., et al., "Tensile properties and fracture locations of friction-stir welded joints of 6061-T6 aluminum alloy”, Journal Of Materials Science, v. 22, pp. 1061-1063, 2003.

[4] MISHRA R.S., MA Z.Y. "Friction stir welding and processing", Materials Science and Engineering: R: Reports, v. 50, n. 1-2, pp. 1-78, Ago. 2005.

[5] THREADGILL, P.L., LEONARD, A.J., SHERCLIFF, H.R., et al., "Friction stir welding of aluminium alloys", $\quad$ http://www.twi-global.com/technical-knowledge/published-papers/friction-stir-welding-ofaluminium-alloys

[6] RODRIGUEZ, R.I., JORDON, J.B., ALLISON, P.G., et al., "Microstructure and mechanical properties of dissimilar friction stir welding of 6061-to-7050 aluminum alloys", Materials \& Design, v. 83, pp. 60-65, Oct. 2015.

[7] MOREIRA, P.M.G.P., SANTOS, T., TAVARES, S.M.O., et al., "Mechanical and metallurgical characterization of friction stir welding joints of AA6061-T6 with AA6082-T6", Materials and Design, v. 30, pp. 180-187, 2009.

[8] STEPHEN LEON, J., JAYAKUMAR, V., "Investigation of mechanical properties of aluminium 6061 alloy friction stir welding", American Journal of Mechanical Engineering and Automation , v. 1, n. 1, pp. 69, 2014.

[9] RODRÍGUEZ, C., GARCÍA, J., CÁRDENAS, E., et al., "Mechanical Properties Characterization of Heat-Affected Zone Using the Small Punch Test”, American Welding Journal, v. 88, pp. 188-192, 2009.

[10] TUFARO, L.N., SVOBODA, H.G., "Efecto del procedimiento de soldadura por FSW en la evolución temporal de las propiedades en uniones de AA 7075-T651", Soldagem \& Inspeção, v. 19, n. 4, pp. 333-342, Oct./Dec. 2014.

[11] TUFARO, L.N., MANZONI, I., SVOBODA, H.G., "Effect of Heat Input on AA5052 Friction Stir Welds Characteristics", Procedia Materials Science, v. 8, pp. 914-923, 2015. 
[12] STATHERS, P.A., HELLIER, A.K., HARRISON, R.P., et al., "Hardness-tensile property relationships for HAZ in 6061-T651 aluminum”, Welding Journal, v. 93, n. 8, pp. 301-311, 2014.

[13] GARCÍA, T., RODRÍGUEZ, C., BELZUNCE, F., et al., "Estimation of the mechanical properties of metallic materials by means of the small punch test", Journal of Alloys and Compounds, n. 582, pp. 708-717, 2014.

[14] MISHRA, R.S., KUMAR, P., Friction Stir Welding and Processing, Springer, 2014. 